\title{
Lymph node cancer of the mediastinum with a putative necrotic primary lesion in the lung: a case report
}

\author{
Daichi Shikata ${ }^{1}$, Takahiro Nakagomi ${ }^{1}$, Rumi Higuchi ${ }^{1}$, Yujiro Yokoyama ${ }^{1}$, Toshio Oyama $^{2}$ and Taichiro Goto ${ }^{1 *}$ (D)
}

\begin{abstract}
Background: Although mediastinal lymph node cancer is presumed to originate in the lung, the primary site is usually unidentified, so the pathological course remains unclear. We recently encountered a case of mediastinal lymph node cancer having a putative primary lesion remaining in the lung as a necrotic focus.
\end{abstract}

Case presentation: The patient was a 56-year-old man who visited our department because computed tomography screening had revealed a nodular shadow in the lingular segment. However, on positron emission tomography, fluorine18 deoxyglucose accumulation was detected in a subcarinal lymph node and not in the nodule in the lingular segment. Biopsy of the lung tumor and the lymph node was performed via minimal thoracotomy. Intraoperative pathologic examination showed necrosis alone and no malignant findings in the lung tumor. By contrast, carcinoma was detected in the lymph node. Additional subcarinal lymph node dissection was performed. Results of postoperative histopathologic examination indicated poorly differentiated adenocarcinoma of the subcarinal lymph node. Meanwhile, the nodule in the lingular segment was speculated to be a spontaneously resolved primary focus of lung cancer.

Conclusions: In this case, the primary lung cancer focus resolved spontaneously after lymph node metastasis, explaining the pathogenesis underlying mediastinal lymph node cancer of unknown primary site. For similar cases of malignancy, aggressive treatment, including surgery, is effective.

Keywords: Mediastinal cancer, Lymph node cancer, Unknown primary site, Immunohistology, Surgery

\section{Background}

Cancer of unknown primary site (CUP) rarely occurs in the mediastinal lymph node, and its underlying pathology is typically not elucidated $[1,2]$. Many cases of mediastinal lymph node cancer are presumed to arise from the lung, but the primary focus remains essentially unidentified at the time of diagnosis [1,3-5]. Although patients are usually treated by chemoradiation therapy or surgery, no standard treatment has been established. We recently encountered a case of mediastinal lymph node cancer with a putative necrotic primary lesion in the lung.

\section{Case presentation}

The patient was a 56-year-old man in whom a nodular shadow was found in the left lower lung field via chest

\footnotetext{
* Correspondence: taichiro@1997.jukuin.keio.ac.jp

1 Department of General Thoracic Surgery, Yamanashi Central Hospital,

Yamanashi 400-8506, Japan

Full list of author information is available at the end of the article
}

radiographic screening. Because close examination via computed tomography $(\mathrm{CT})$ revealed a nodular shadow in the left lingular segment, the patient was referred to our department for surgery. He was an active smoker who consumed 1 pack/day for 30 years. No other noteworthy features were found in his past history or physical findings. The CT scan showed a $1-\mathrm{cm}$ nodule in the left lingular segment (Fig. 1). However, positron emission tomography revealed fluorine-18 deoxyglucose (FDG) accumulation in the subcarinal lymph node and not in the nodular shadow in the lingular segment (Fig. 2a, b). FDG uptake was not noted in other organ lesions. Serum carcinoembryonic antigen (CEA) was elevated at $14.3 \mathrm{mg} / \mathrm{ml}$ (normal range $0.0-5.0 \mathrm{ng} / \mathrm{ml}$ ). Biopsy of the lesions in the lung and the lymph node was performed for a definite diagnosis.

The patient underwent thoracoscopic surgery via two incisions by video-assisted thoracoscopic approaches: a $12-\mathrm{mm}$ camera port incision was made at the eighth 


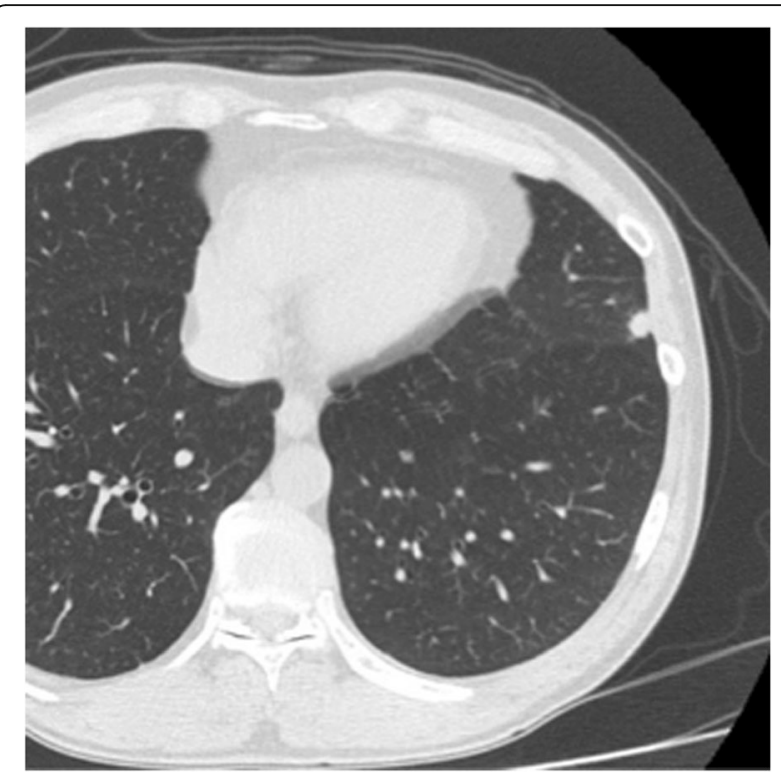

Fig. 1 Computed tomography. A small nodule was found in the lingular segment

intercostal space, the posterior axillary line; and a 4-cm incision at the fourth intercostal space, the anterior axillary line. At the time of surgery, a 1-cm nodule was palpated in the lingular segment, and wedge resection at that site was performed. Intraoperative pathologic examination revealed inflammation and necrosis. The subcarinal lymph nodes were also resected and examined pathologically. Because the results indicated a cancer lesion, the remaining subcarinal and hilar lymph nodes were dissected. The patient was diagnosed with mediastinal lymph node cancer, and no additional lung resection, such as left upper lobectomy, was performed.

Histopathologic examination of the intrapulmonary nodule revealed necrosis and no viable tumor cells inside (Fig. 3a, b). Alveolar elastic fibers were well-maintained, and veins full of necrotic cells were observed (Fig. 3c, d). Granuloma was not evident, and acid-fast staining provided no evidence of acid-fast bacteria. Immunostaining showed irregular features positive for pan-cytokeratin
(AE1/AE3) and napsin A in the necrotic tissue, suggesting residual cancer tissue (Fig. $4 \mathrm{a}, \mathrm{b})$. Meanwhile, a proliferation of large cancer cells with eosinophilic cytoplasm was found in the lymph node specimen (Fig. 5a, b). Cancer cells were found in the subcarinal lymph node, but not in additionally dissected regional lymph nodes. Immunostaining of the tumor cells was positive for pan-cytokeratin, TTF-1, and napsin A but negative for $\mathrm{p} 40$ and $\mathrm{p} 63$, suggesting a poorly differentiated adenocarcinoma of pulmonary origin (Fig. 5 c, d).

The postoperative course was favorable, and the patient was discharged on the fifth postoperative day. Given that the cancer was at pTxN2M0 and stage IIIA according to lung cancer staging, concurrent chemoradiotherapy comprising systemic chemotherapy (cisplatin and vinorelbine) and radiotherapy of $60 \mathrm{~Gy} / 30 \mathrm{Fr}$ was administered. The serum CEA level reverted to normal after surgery (Fig. 6). To date, at 3 years after surgery, no recurrence has been noted.

\section{Discussion}

CUP is the collective term for a group of cancers for which the anatomical site of origin remains unidentified after a metastatic focus is found [6]. CUP is characterized by clinically unconfirmed primary malignancy, early occurrence of dissemination, rapid progression, and difficult prediction of the metastatic pattern $[2,6]$. The incidence of CUP is reported to be approximately 0.5-6. $7 \%$ [7-10]. It occurs more frequently in men than in women, and most frequently involves the lymph nodes and bones [10]. Histologically, most cases are adenocarcinomas [10]. CUP rarely occurs in mediastinal lymph nodes, accounting for only $1.0-1.5 \%$ of all of the CUP cases $[9,10]$.

A possible pathogenesis of lymph node CUP is a small primary focus that cannot be detected with diagnostic imaging [3] or spontaneous resolution of the primary focus $[10,11]$. Some immunologic mechanisms may be involved in the spontaneous resolution of the primary focus $[11,12]$. Lymph node cancer of unknown primary origin possibly occurs if the primary focus is resolved
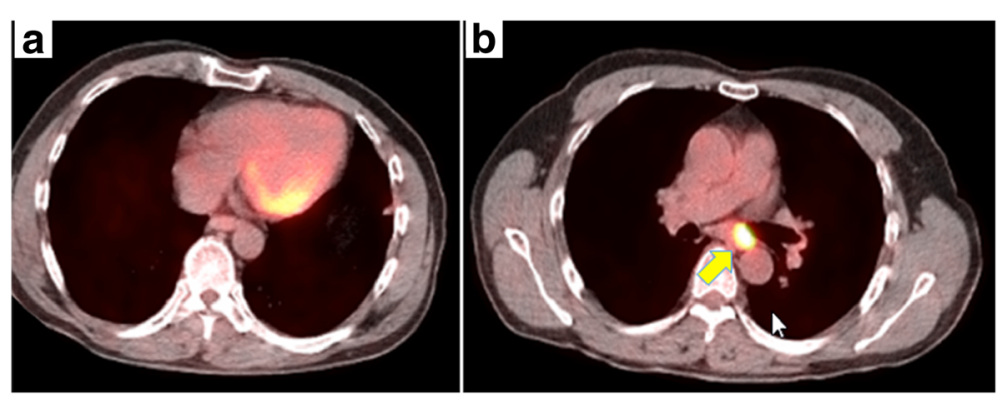

Fig. 2 Positron emission tomography. a, b FDG uptake was detected in a subcarinal lymph node and not in a nodule in the lingular segment. The arrow indicates the lymph node with FDG accumulation 


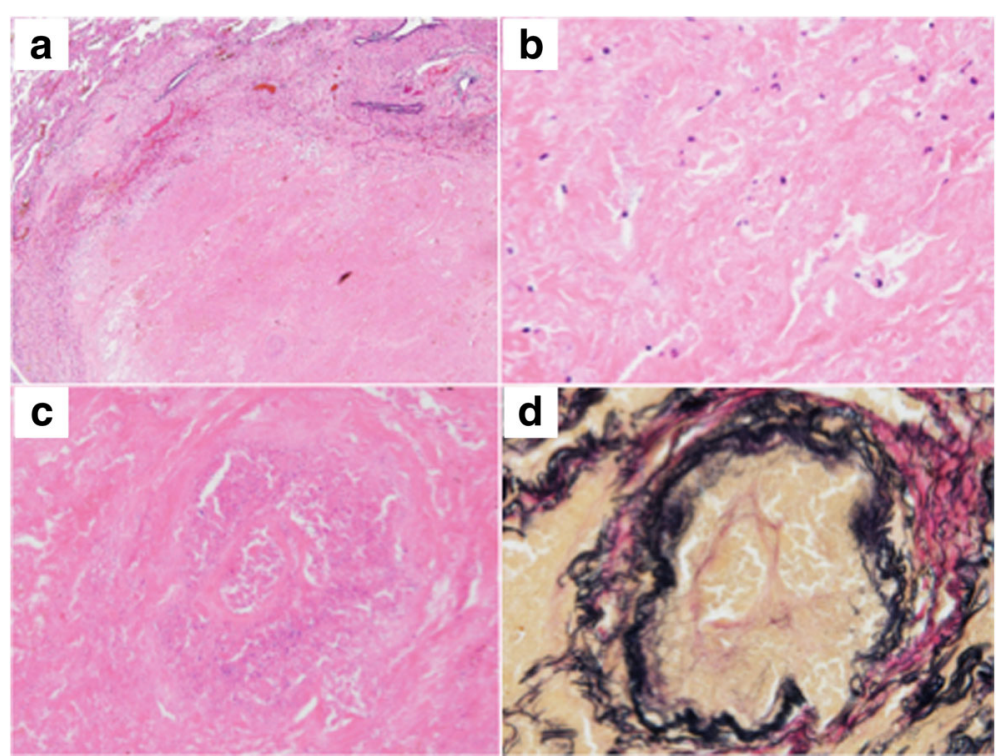

Fig. 3 Histopathologic findings of the pulmonary nodule. $\mathbf{a}$, b No viable cells could be observed inside the tumor (hematoxylin and eosin stain. a low magnification, b high magnification). c, d Vein filled with necrotic cells were observed (c hematoxylin and eosin stain; d Elastica van Gieson stain)

spontaneously by host immunity, while a metastatic lymph node evades immune reactions. On the other hand, the lymph node itself may be the primary focus. In the latter case, malignant transformation of the ectopic epithelium in the lymph node may be responsible [10, 13-15]. However, all of these mechanisms are hypothetical and not based on scientific evidence.

In approximately $40 \%$ of reported cancers in the hilus, mediastinum, and cervical lymph nodes, the site of primary malignancy was the lung [10]. Taking into account the pathway of regional lymph flow in the lung, occult microcarcinoma can be considered to be present in the lung in cases of mediastinal lymph node CUP. Therefore, mediastinal lymph node cancer is commonly treated based on the assumption that the primary focus is lung cancer. In general, CUP prognosis is poor, with a median survival period of 2-9 months and a 5-year survival rate of $2.8-6.0 \%[2,8]$. Meanwhile, mediastinal lymph node CUP follows a clinical course different from that of CUP in general, and the prognosis is favorable when the localized focus in the lymph node is resected $[3,5,16]$. In cases of mediastinal lymph node enlargement, a surgical approach should be considered for both diagnostic and therapeutic purposes, even if the primary focus cannot be identified.

In our case, the mechanism of metastasis can be explained by lymphatic metastasis along the regional lymph flow, assuming that the primary focus was the nodule in the lingular segment and that the metastatic site was a subcarinal lymph node. The positive pancytokeratin and napsin A staining and the presence of necrotic cells in the blood vessels indicated the previous existence of cancer at that pulmonary site. There was no recurrence, including new primary lesions, during the 3-year follow-up after surgery noted in our patient. Thus, lymph node metastasis originating from lung cancer was highly probable based on the above reasons, although a definitive diagnosis is not possible. We

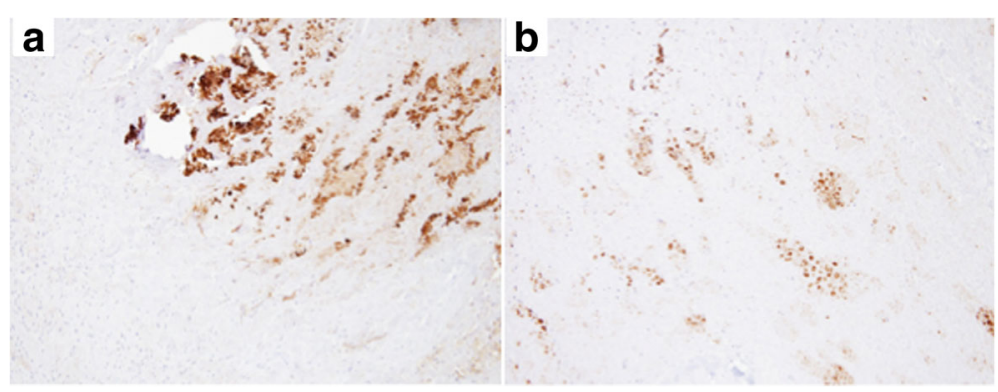

Fig. 4 Immunohistochemical findings of the pulmonary nodule. Immunostaining results of the necrotic tissue were positive for pan-cytokeratin (AE1/AE3) and napsin A (a pan-cytokeratin immunostaining; b napsin A immunostaining) 


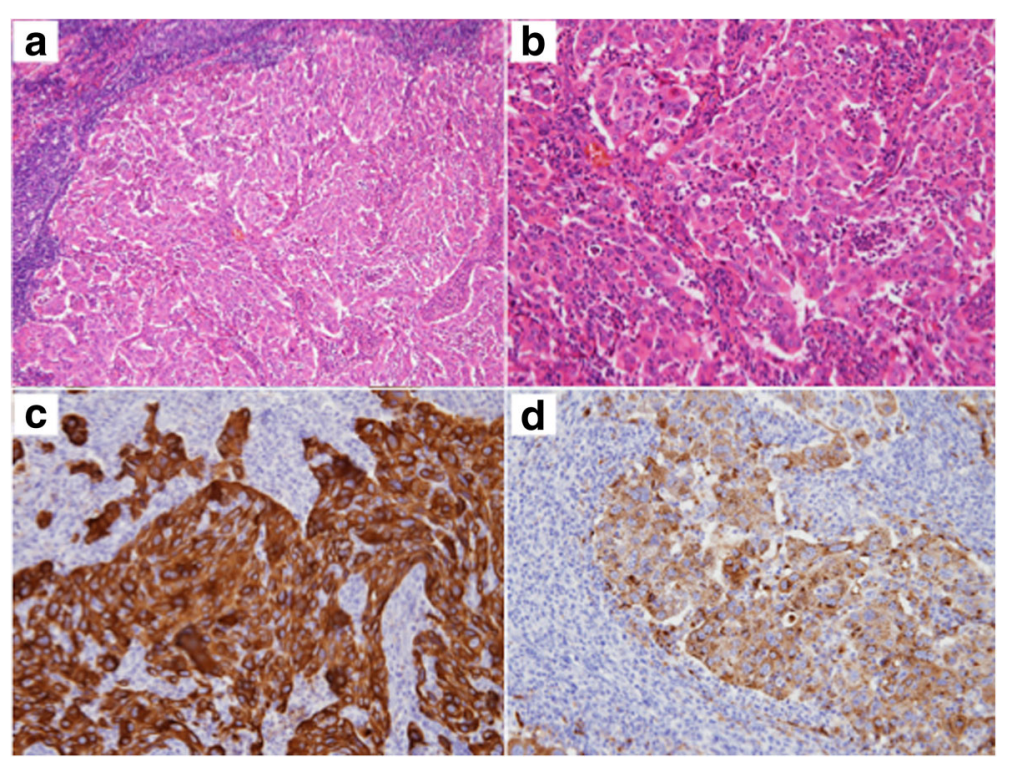

Fig. 5 Histopathologic findings of the subcarinal lymph node. $\mathbf{a}$, b Solid proliferation of cancer cells was observed (hematoxylin and eosin stain. a low magnification, b high magnification). c, d Tumor cells were positive for pan-cytokeratin and napsin A (c pan-cytokeratin immunostaining; d napsin A)

believe that this case of mediastinal lymph node cancer exemplifies the hypothesis that a primary focus of lung cancer becomes necrotic through an immunologic mechanism during the clinical course, and a metastatic focus in the mediastinal lymph node alone survives and grows. Kohdono et al. reported a case of an increased metastatic focus in the mediastinal lymph node, with the primary lung cancer resolving spontaneously during the clinical course [4]. Although our present case resembles their case in some respects, to our knowledge, this is the first case of a pathologically verified etiologic mechanism of mediastinal lymph node cancer with the primary lesion remaining as necrotic tissue in the lung.

Recently published case reports regarding cancer of the intrathoracic lymph nodes were reviewed $[3,4,16-$ 25]. The patient characteristics of all 18 cases (including our case) are shown in Table 1 . Fifteen patients were

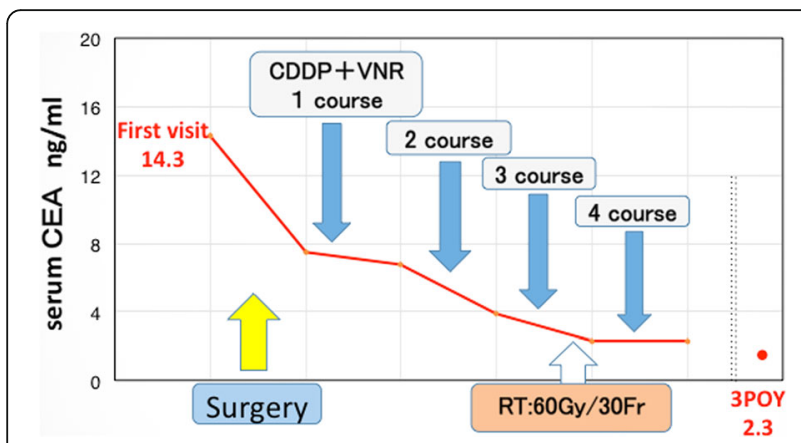

Fig. 6 Time-course changes in the serum CEA level. The abscissa denotes time, and 1 scale corresponds to 1 month. Parallel dotted lines indicate omission of an intermediate step men and three were women; their average age was 63. 5 years (range, 40-83 years). Except for one female patient, all patients were smokers. Seven patients had adenocarcinomas, six had squamous cell carcinomas, three had large cell carcinomas, and one had a small cell carcinoma. In 13 patients, certain tumor markers exceeded the normal range. The CUP was located in the lymph nodes of the mediastinum in 14 cases and in the pulmonary hilum in 4 cases. Surgery was performed in 13 patients, and adjuvant treatment after CUP resection consisted of radiation therapy in 4 cases, chemoradiation therapy in 3 cases, and no additional treatment in 6 cases. The outcomes after the treatment were as follows: 15 patients remained alive without recurrence or disease progression at an average of 28.8 months (range, 382 months), two patients remained alive with recurrence at an average of 66 months (6 and 126 months), and one patient died because of the disease 6 months after the treatment.

Completely resected mediastinal lymph node CUP reportedly has a better prognosis than lung cancer with mediastinal lymph node metastasis $[4,10]$. Complete resection is the first-line treatment if the lesion of the lymph node cancer of the hilum or mediastinum is localized $[3,5,16]$. Although the lung is highly likely to be the primary site of metastatic mediastinal lymph node cancer, lobectomy is generally avoided to preserve respiratory function. Even though chemoradiotherapy is often adopted as a postoperative treatment modality, there is no consensus on its therapeutic efficacy. In this case, postoperative chemoradiotherapy was performed at the request of the patient. 


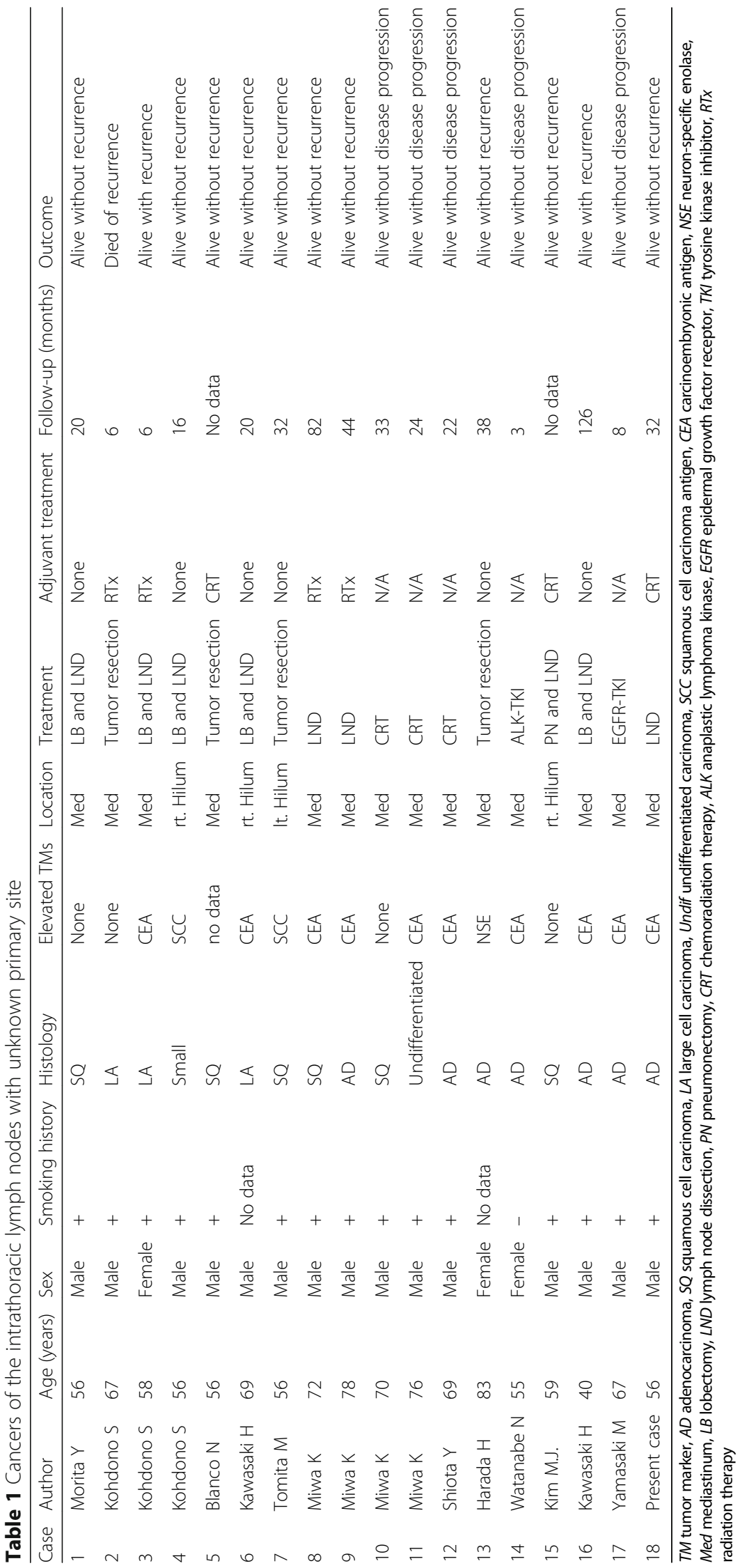




\section{Conclusion}

This case shows that lymph node CUP may occur due to the regression of the carcinoma itself at the primary site. As such, aggressive treatment, including surgical resection, should be performed for mediastinal lymph node CUP.

\section{Abbreviation}

CEA: Carcinoembryonic antigen; CT: Computed tomography; CUP: Cancer of unknown primary site; FDG: Fluorine-18 deoxyglucose

\section{Acknowledgements}

Not applicable.

\section{Funding}

No funding was received for this study.

\section{Availability of data and materials}

All data generated or analyzed during this study are included in this published article.

\section{Authors' contributions}

DS and TG wrote the manuscript. DS, TN, RH, YY, and TG performed the surgery. DS and TO performed the pathologic examination. TG was involved in the final editing of the manuscript. All authors read and approved the final manuscript.

\section{Ethics approval and consent to participate}

The study was approved by the institutional ethics board of Yamanashi Central Hospital.

\section{Consent for publication}

Written informed consent was obtained from the patient for publication of this case presentation and accompanying images. A copy of the written consent is available for review by the Editor-in-Chief of this journal.

\section{Competing interests}

The authors declare that they have no competing interests.

\section{Publisher's Note}

Springer Nature remains neutral with regard to jurisdictional claims in published maps and institutional affiliations.

\section{Author details}

'Department of General Thoracic Surgery, Yamanashi Central Hospital, Yamanashi 400-8506, Japan. ${ }^{2}$ Department of Pathology, Yamanashi Central Hospital, Yamanashi, Japan.

Received: 29 November 2017 Accepted: 21 March 2018

Published online: 02 April 2018

\section{References}

1. Izumi Y, Mukai M, Kikuchi K, Kobayashi K. Long-term survival after incomplete resection of immunohistochemically diagnosed TON1 lung cancer: report of a case. Surg Today. 2006;36:270-3.

2. Pavlidis N, Briasoulis E, Hainsworth J, Greco FA. Diagnostic and therapeutic management of cancer of an unknown primary. Eur J Cancer. 2003;39:1990-2005.

3. Kawasaki H, Arakaki K, Taira N, Furugen T, Ichi T, Yohena T, Kawabata T. Lung cancer detected 5 years after resection of cancer of unknown primary in a mediastinal lymph node: a case report and review of relevant cases from the literature. Ann Thorac Cardiovasc Surg. 2016;22:116-21.

4. Kohdono S, Ishida T, Fukuyama Y, Hamatake M, Takenoyama M, Tateishi M, Sugimachi K. Lymph node cancer of the mediastinal or hilar region with an unknown primary site. J Surg Oncol. 1995;58:196-200.

5. Yoshizu A, Kamiya K. Mediastinal lymph node carcinoma of unknown primary site; report of a case. Kyobu Geka. 2012;65:507-9.

6. Briasoulis E, Pavlidis N. Cancer of unknown primary origin. Oncologist. 1997: 2:142-52.
7. Fizazi K, Greco FA, Pavlidis N, Pentheroudakis G, Group EGW. Cancers of unknown primary site: ESMO Clinical Practice Guidelines for diagnosis, treatment and follow-up. Ann Oncol. 2011;22(Suppl 6):vi64-8.

8. Greco FA, Burris HA 3rd, Erland JB, Gray JR, Kalman LA, Schreeder MT, Hainsworth JD. Carcinoma of unknown primary site. Cancer. 2000;89: 2655-60

9. Holmes FF, Fouts TL. Metastatic cancer of unknown primary site. Cancer. 1970;26:816-20.

10. Riquet M, Badoual C, le Pimpec BF, Dujon A, Danel C. Metastatic thoracic lymph node carcinoma with unknown primary site. Ann Thorac Surg. 2003;75:244-9.

11. Cafferata MA, Chiaramondia M, Monetti F, Ardizzoni A. Complete spontaneous remission of non-small-cell lung cancer: a case report. Lung Cancer. 2004:45:263-6.

12. So T, Takenoyama M, Ichiki Y, Mizukami M, So T, Hanagiri T, Sugio K, Yasumoto K. A different pattern of cytotoxic T lymphocyte recognition against primary and metastatic tumor cells in a patient with nonsmall cell lung carcinoma. Cancer. 2005;103:200-8.

13. Fellegara G, Carcangiu ML, Rosai J. Benign epithelial inclusions in axillary lymph nodes: report of 18 cases and review of the literature. Am J Surg Pathol. 2011:35:1123-33.

14. Gould VE, Warren WH, Faber LP, Kuhn C, Franke WW. Malignant cells of epithelial phenotype limited to thoracic lymph nodes. Eur J Cancer. 1990;26:1121-6.

15. Ishimaru Y, Shibata Y, Ohkawara S, Ohshima H, Kihara S. Lymphoepithelial cystic lesion related to adenocarcinoma in the mediastinum. Am J Clin Pathol. 1989;92:808-13.

16. Harada $H$, Yamashita $Y$, Kuraoka K, Taniyama K. Sequential mediastinal lymphadenectomy of an unknown primary tumor. Ann Thorac Surg. 2013;95:687-9.

17. Blanco N, Kirgan DM, Little AG. Metastatic squamous cell carcinoma of the mediastinum with unknown primary tumor. Chest. 1998;114:938-40.

18. Kawasaki H, Yoshida J, Yokose T, Suzuki K, Nagai K, Hojo F, Kodama T, Nishiwak Y. Primary unknown cancer in pulmonary hilar lymph node with spontaneous transient regression: report of a case. Jpn J Clin Oncol. 1998:28:405-9.

19. Kim MJ, Lim SH, Han SJ, Choi KH, Lee SH, Park MW, Kang H, Na JO. Indolent metastatic squamous cell carcinoma of unknown primary in the intrathoracic lymph node: a case report and review of the literatures. Tuberc Respir Dis (Seoul). 2015;78:23-6.

20. Miwa K, Fujioka S, Adachi Y, Haruki T, Taniguchi Y, Nakamura H. Mediastinal lymph node carcinoma of an unknown primary site: clinicopathological examination. Gen Thorac Cardiovasc Surg. 2009;57:239-43.

21. Morita Y, Yamagishi M, Shijubo N, Nakata H, Kurihara M, Asakawa M. Squamous cell carcinoma of unknown origin in middle mediastinum. Respiration. 1992;59:344-6.

22. Shiota Y, Imai S, Sasaki N, Tahara K, Noma B, Horita N, Taniguchi A, Ono T. A case of mediastinal lymph node carcinoma of unknown primary site treated with docetaxel and cisplatin with concurrent thoracic radiation therapy. Acta Med Okayama. 2011;65:407-11.

23. Tomita M, Matsuzaki Y, Shimizu T, Hara M, Ayabe T, Enomoto Y, Onitsuka T. Squamous cell carcinoma of the hilar lymph node with unknown primary tumor: a case report. Ann Thorac Cardiovasc Surg. 2008;14:242-5.

24. Watanabe N, Ishii T, Takahama T, Tadokoro A, Kanaji N, Dobashi H, Bandoh S. Anaplastic lymphoma kinase gene analysis as a useful tool for identifying primary unknown metastatic lung adenocarcinoma. Intern Med. 2014;53:2711-5.

25. Yamasaki M, Funaishi K, Saito N, Sakano A, Fujihara M, Daido W, Ishiyama S, Deguchi N, Taniwaki M, Ohashi N, Hattori N. Putative lung adenocarcinoma with epidermal growth factor receptor mutation presenting as carcinoma of unknown primary site: a case report. Medicine (Baltimore). 2018;97:e9942 